\title{
Editorial
}

\section{Neo liberalización y Ciudadanía(s) en el Sur Global}

\author{
Hernán Cuevas \\ Universidad de Concepción, Concepción, Chile \\ Email: hcuevasv@udec.cl \\ Felipe González \\ Universidad Central de Chile, Santiago, Chile. \\ Email: felipe.gonzalez@ucentral.cl \\ Juan Pablo Paredes \\ Universidad de los Lagos, Santiago, Chile \\ Email: juan.paredes2@ulagos.cl
}

\section{Introducción}

La crítica del neoliberalismo y la consiguiente afirmación de la ciudadanía con sus demandas por derechos sociales, justicia y reconocimiento, han ocupado un lugar central en los debates en la esfera pública desde la irrupción de las protestas sociales en escala global (Della Porta, 2015; Ancelovici, Dufour\&Nez, 2016), con un especial ímpetu en el Sur Global (Bringel\&Pleyers, 2017). Un caso ejemplar fue el del ciclo de protestas por la educación en Chile a partir de 2011. Por ejemplo, los estudiantes chilenos no sólo lograron posicionar la educación por medio de su lucha como el más notorio issue político en la agenda nacional, sino que además lograron redefinir su significado, desplazando el sentido dominante e institucionalizado de educación como bien de consumo (Bellei, Cabalín\& Orellana, 2014; Fleet\& Guzmán Concha, 2016; Donoso, 2017; Cuevas \& Paredes, 2018; DisiPavlic, en Prensa). Junto con demandar educación gratuita y de calidad, los estudiantes instalaron la idea de la educación como un derecho social y exigieron poner fin al lucro en educación. La difusión e impacto de este contra-discurso que criticó los fundamentos de la hegemonía neoliberal resonó más allá de las fronteras nacionales y fue internacionalmente reconocido, llegando a ser imitado en otras regiones como el caso Canadiense del 2012. Si bien este proceso se encuentra inconcluso, pues la lucha de posiciones prosigue con el actual periodo presidencial (2018-2022), este movimiento y la amplia coalición social que logró forjar en torno a sus demandas contribuyó a abrir una ventana de oportunidades para introducir cambios importantes en la sociedad chilena. 
El movimiento de los estudiantes chilenos es un caso ejemplar que, lejos de constituir un accidente aislado, forma parte de un contexto regional más amplio de efervescencia y protesta social contra las políticas neoliberales implementadas por gobiernos con variadas bases de apoyo. ${ }^{1} \mathrm{En}$ efecto, en varios países de América Latina sectores sociales populares, comunidades indígenas, y movimientos socio ambientales y socio territoriales se han opuesto a megaproyectos de inversión, sean estos de carácter público, privado, o de una alianza público-privada. Varios de estos megaproyectos promueven operaciones capitalistas extractivas a gran escala, principalmente en los sectores de minería, hidrocarburos, pesca, agroindustria y forestal (Cuevas, Julián \& Rojas (en prensa), De La Fuente, Ricaldi\&Saldomando (eds.) 2017, Schmalz\& Ramírez (eds.) en prensa). También los megaproyectos de inversión energéticos, infraestructura logística y de transporte, cuyo propósito es frecuentemente crear las condiciones o apoyar las operaciones extractivas, han encontrado resistencias (Budrovich\& Cuevas 2018, Cuadra 2018).

Los significantes “ciudadanía” y “neoliberalismo” no sólo han sido movilizados por varios contra-discursos y movimientos de protesta en América Latina, sino que también forman parte de las categorías centrales del discurso crítico de una nueva intelectualidad latinoamericana crítica (cf. Escobar 2010, Svampa 2015, 2017, Santos 2002; García Linera, 2008). Así, en varios discursos políticos y teóricos que circulan en la esfera pública se aprecia una tensión entre la idea de ciudadanía como fundamento de un régimen de derechos, justicia social y democracia, y las políticas económicas neoliberales inspiradas en el monetarismo y la teoría económica neoclásica y sus efectos de mercantilización de la sociedad que limitaría o afectaría los derechos (Escobar 2003, 2010, Santos 2002, García Linera, 2008).

En el Norte Global, por su parte, la importancia en el discurso teórico de la tensión entre ideas de ciudadanía y neoliberalismo es también relevante, en especial en los debates sobre la crisis de representación, la postdemocracia, el ascenso de la derecha populista, y la creciente precariedad y desigualdad social (Crouch 2004, Errejón y Mouffe 2015, Standing 2011, 2014). Junto a estas controversias político intelectuales, los debates polìticos que dominaron los más recientes procesos eleccionarios en países como Alemania, Francia y Austria también reflejan la misma tensión entre neoliberalismo y ciudadanía. Aunque de manera más tangencial, también en los casos del denominado Brexit, del debate (y referéndum) por la independencia en Cataluña, e incluso en la polémica entre el presidente Trump y los deportistas de color en US que culminó en las manifestaciones por la supremacía blanca en el Estado de Virginia, también presentan la misma tensión entre ciudadanía y neoliberalismo.

No obstante lo anterior, cabe preguntarse si en verdad son ciudadanía y neoliberalismo categorías incompatibles, e incluso contrapuestas. ¿Qué efectos ha producido sobre la ciudadanía la instauración y consolidación del neoliberalismo? ¿Es acaso el neoliberalismo un régimen socioeconómico que ha echado por tierra no sólo los derechos sociales como institución, 
sino incluso su concepto y tal vez también el imaginario de una ciudadanía más sustantiva? O, al contrario, neoliberalismo y ciudadanía pueden ser compatibles a condición que los contenidos de la ciudadanía y/o las políticas neoliberales y su implementación sean resignificadas en algún sentido. Este artículo ofrece una reflexión sobre la relación entre ciudadanía y neoliberalismo cuya hipótesis es que la maleabilidad del proyecto neoliberal y los regímenes de ciudadanía concretos ha permitido redefinir sin abolir la institución de la ciudadanía. En el artículo nos concentramos en la situación de Chile y América Latina, e ilustramos nuestro argumento con varios casos de la región, algunos incluso presentes en este mismo volumen, con el propósito de establecer puentes y un diálogo con sus autores en el contexto mayor del debate latinoamericano sobre el neoliberalismo y la ciudadanía.

En las secciones dos y tres desarrollamos brevemente nuestra comprensión de la ciudadanía y el neoliberalismo respectivamente. En la última sección, junto con presentar brevemente los artículos del dossier, sugerimos que lejos de tratarse de categorías irreconciliables, los neoliberalismos y las ciudadanías realmente existentes analizadas en el dossier constituyen buenos ejemplos de las múltiples articulaciones posibles.

\section{La ciudadanía: de la Antigüedad a la Modernidad}

La ciudadanía ha sido conceptualizada de manera explícita desde tiempos remotos (por ejemplo, Aristóteles 1988). La ciudadanía también fue un concepto político importante en la época moderna. Por ejemplo, es central en la teoría política moderna Jean Jacques Rousseau (1977), y se encuentra como trasfondo significativo en ese magnífico manifiesto de Immanuel Kant ¿Qué es la Ilustración? (1994 [1784]). También está presente en la obra de Georg Friedrich Hegel (2000). Y si bien es cierto que Marx (1978) criticó fuertemente los supuestos y eficacia de la ciudadanía, desde entonces su importancia como concepto político fue en ascenso. Su relevancia como idea política moderna estructurante del orden político se encuentra en la base de la difusión en la cultura política democrática y su institucionalización a nivel global. Posiblemente fue durante la Revolución Francesa, y en particular con el hito de la Declaración de los Derechos del Hombre y del Ciudadano (1789), cuando la idea de ciudadanía se consolidó en la cultura política moderna.

La centralidad de la ciudadanía en la época moderna no puede ser simplemente trasplantada a la experiencia de América Latina, al contrario, requiere ser problematizado. En primer término, esta experiencia llegó a suelo americano mediado por la fuerte impronta medieval y escolástica que caracterizó la reflexión acerca de las gentes del nuevo mundo y el carácter del encuentro entre las culturas ibérica y precolombinas durante las fases de descubrimiento y conquista. Sólo en siglos posteriores, con la colonización de América Latina, puede comprenderse la ciudadanía en sus antecedentes, mediaciones conceptuales y traducciones, a partir de los desplazamientos retóricos y los respectivos campos semánticos de los términos 
ciudadano y vecino. A pesar de sus diferencias, ambos términos aluden a un campo semántico común que se refiere a la participación política, a los derechos y obligaciones o deberes, que se expresan en las cargas y los privilegios, beneficios o derechos. Junto con ello, la figura del ciudadano o vecino está siempre en relación a una comunidad -punto retomado más adelante en base al texto de Letelier et alt, en este número.-, la que dependiendo del momento histórico y la coyuntura, era concebida propiamente como una comunidad política (soberana), o simplemente como un agregado de habitantes de una villa, un pueblo o ciudad.

Existe una especie de pre-historia de la idea de ciudadanía en Iberoamérica. Sobre esta pre-historia acerca de los antecedentes de la transformación paradigmática que se sufriría en las esferas públicas latinoamericanas después de 1808, vale la pena citar in extenso uno de los pocos estudios de historia conceptual en la región:

"Ciudadano" es un viejo concepto europeo insertado de modo peculiar en la cultura iberoamericana. Hasta casi 1808 era un concepto inseparable de "vecino". Ciudadano/vecino implicaba un hombre con ciertos privilegios y cargas en el mundo local. Usualmente, en el Antiguo Régimen el término más utilizado era “vecino” y abarcaba a un mayor número de personas. Posteriormente, ciudadano y vecino se iban diferenciando, y sus significados comenzarían a contener cargas semánticas diferentes. (...) En el siglo XIX, “ciudadano” adquiría progresivamente una resonancia nacional e iría dejando, aunque no siempre, el uso de "vecino” para la esfera local. (Aljovin 2009: 180)

La difusión de la idea de ciudadanía, o ciudadano/vecino, en la restringida esfera pública en los territorios latinoamericanos que emergió con mayor fuerza después de las luchas de Independencia tuvo como antecedente la formación de las primeras Juntas de Gobierno locales durante la ocupación napoleónica de la metrópolis española (cf. Forment 2003, Stuven 2000). La revolución mexicana y la formación del PRI, los populismos inclusivos de Perón en Argentina y de Vargas en Brasil, así como el particular socialismo populista e indigenista del APRA en Perú, tuvieron también un importante impacto en la formación de una vaga idea de ciudadanía latinoamericana (cf. Hartlyn\& Valenzuela,1997).

El caso chileno es un buen ejemplo de un estable y continuo pero a la vez lento patrón de inclusión de las masas al sistema político. Las elecciones de 1920, junto a la movilización social que las precedió y acompañó, constituyen el mayor hito en el desarrollo de la ciudadanía política en la emergente democracia de masas (Millar 1982). Así, el desarrollo de la ciudadanía democrática en Chile fue más bien restrictivo y controlado (Barros y Vergara 1991, Valenzuela y Valenzuela 1983, Valenzuela y Hartlyn\& Valenzuela, 1997, Maza 1995, Moulián 1993, Gamboa 2011), que un elemento universalizable o fácilmente generalizable. Junto con el rol de los partidos progresistas de izquierda, la inclusión fue también promovida en Chile por los Conservadores, quienes veían en el voto rural y femenino un importante 
caudal de apoyos (Maza 1995). Otros momentos importantes en la formación de las ciudadanías latinoamericanas, más allá de la dimensión política y civil en ella, se relaciona con la demanda "de inclusión social y de igualdad de condiciones materiales” de varios sectores de la población ligados al mundo del trabajo y del mundo urbano-popular, a inicios del siglo XX y en contextos en procesos de urbanización creciente, en lo que se ha llamado la cuestión social (Grez Toso, 1997). Las presiones de vastos sectores de la población ligada al mundo popular y a la clase obrera relacionadas con las precarias y deplorables condiciones materiales en las que transcurría sus vidas, que terminan en expresiones colectivas y masivas de protesta (Grez Toso, 1997; Garcés, 2012), adelantan en Chile y América Latina debates posteriores sobre la ciudadanía en su alcance social.

La concepción moderna de la ciudadanía se basa en la figura de un status legal que es reconocido al individuo bajo la autoridad de un estado. Esta comprensión ha sido criticada para ampliar sus dimensiones civil y política, y de este modo incluir varias dimensiones sociales y culturales negadas o excluidas que se relacionan a las luchas políticas y sociales por el reconocimiento y/o la redistribución como instancias de reivindicación o demanda y, por ende, de ciudadanía. Por su parte, los actores contestatarios y sus luchas basadas en la identidad y la diferencia, sean éstas sexuales, raciales, diaspóricas, étnicas-nacionales, o territoriales y ecológicas, han encontrado nuevas formas de articular sus demandas como reclamos de ciudadanía. De este modo, la ciudadanía dejó de ser entendida sólo como un estatus legal y, junto con ello, para ser concebida contemporáneamente también como una categoría y una práctica social de reconocimiento político y social, y de redistribución económica (Isin y Turner 2002, 2007, Isin y Wood 1999).

En la teoría política y social contemporánea, la ciudadanía ha cobrado especial importancia (Kymlicka y Norman 1994). Esta mayor notoriedad de la categoría ha venido de la mano del auge de los debates sobre la democracia y las diferentes teorizaciones que de ella se han elaborado (democracia agregativa o schumpeteriana, democracia deliberativa, democracia participativa, democracia radical, etc.), del feminismo, del renacer contemporáneo del republicanismo, y los debates sobre el multiculturalismo, las políticas de identidad y las diferentes aproximaciones a los nuevos movimientos sociales.

Desde la teoría social también han surgido un conjunto de trabajos que dan centralidad a la categoría de ciudadanía, especialmente relacionados con los debates de la globalización y el descentramiento de la idea de nación, con el surgimiento de formas de vida transnacionales ligados a procesos de migración, lo que ha planteado desafíos a la resignificación de la idea de ciudadanía (Santos y Rodríguez, 2007; Sassen, 2015; Ramírez, 2016). En América Latina, así como en todo el Sur Global, estas discusiones se tiñen con tintes locales al entrar en diálogo con las cuestiones sobre el neoextractivismo, las discusiones acerca del posdesarrollo y las perspectivas decoloniales y subalternistas (Escobar 2010; Svampa, 2017). 
El debate sobre ciudadanía indígena y el multiculturalismo en América Latina ofrece un buen ejemplo de esta recepción activa. A pesar que ha habido importantes avances de las minorías étnico-raciales e indígenas en términos de reconocimientos simbólico, cultural e incluso político en varios países de América Latina, el caso de los pueblos indígenas es particularmente ilustrativo de los arreglos ambivalentes a los que se ha llegado. En efecto, varios estudios han observado que junto con el reconocimiento del multiculturalismo, en el marco de la expansión de los sectores extractivos en el continente, sea bajo regímenes neoliberales o de los nuevos progresismos, la retórica del reconocimiento no se ha traducido en formas de devolución o redistribución del poder que benefician a los pueblos indígenas (Cuadra 2014, Hale, 2007; Richards, 2013).

Pero la relevancia de la ciudadanía no está confinada a las reflexiones de la teoría social y política. En efecto, la ciudadanía ha cobrado importancia creciente como temática de estudio en la sociología política y la ciencia política empírica (Janoski 1998, Klingeman y Fuchs (eds.) 1998; 2002, Nash 2010). Todas estas corrientes, muy dispares en intereses y perspectivas teóricas, han contribuido para que en décadas recientes emergiera y luego se haya consolidado un campo de estudios interdisciplinario y/ o transdisciplinario en torno de la ciudadanía (cfr. la revista CitizenshipStudies, Isin y Turner (eds.) 2001, 2007). A pesar de estos importantes avances, este campo no se haya consolidado, y en nuestra región no es raro encontrar una situación de fragmentación disciplinaria, en la que las distintas perspectivas funcionan como "mesas separadas".

Por otra parte, la variedad de enfoques disciplinarios ha multiplicado las definiciones disponibles de ciudadanía. Como consecuencia, uno de los problemas que enfrentamos al estudiar la ciudadanía es su multivocidad y su ambigüedad conceptual. Ciertamente, ciudadanía es un concepto debatido (Isin, 2009), pero junto al problema de la vaguedad y ambigüedad de la “ciudadanía”, nos encontramos con otro problema inherente al concepto: su ambivalencia (cfr. Bauman 1991, Junge, 2008). Por ambivalencia se entiende la presencia constituyente de principios y lógicas opuestas: por una parte, una dimensión de control y gobierno inherente a la ciudadanía -es decir, la ciudadanía como dispositivo de poder para el gobierno de las poblaciones-; y, por otra parte, una dimensión emancipatoria o crítica, que ha servido históricamente para liberar a extensas clases sociales y grupos subalternos de diferentes formas de opresión. Tal ambivalencia dota de cierta incertidumbre al concepto y permite proponer nuevas orientaciones.

La mayor parte de la literatura especializada admite que la ciudadanía incluye los siguientes dos factores: un estatuto de pertenencia a una comunidad política y/o y un sistema de derechos y deberesde las personas (Isin y Wood, 1999; Janoski, 1998; Marshall, 1997). Ambos aspectos se refieren a la ciudadanía de manera restringida, como una institución política que relaciona al individuo con el estado (Janoski, 1998). De lo anterior se deriva como tercer elemento el estado-nación, que ha sido el ambiente a la vez cultural y territorial en el que se desarrolló la ciudadanía moderna (Barbalet, 
2007). Considerados conjuntamente, estos 3 factores constituyen el corazón de la comprensión jurídico-política y minimalista de la ciudadaníaque domina en buena parte de la literatura de la ciencia política (ver Dalton, 2006; Klingeman y Fuchs (eds.) 1998; 2002) y en algunos enfoques de la sociología política (ver Janoski, 1998). Sin embargo, debido a su reducida intensiónconceptual (Gerring y Baresi, 2003) proponemos que este concepto mínimo y jurídico- político de ciudadanía debe ser expandido para permitirnos comprender algunos de los procesos más dinámicos que influyen actualmente en la expansión de la ciudadanía realmente existente.

La literatura clásica sobre ciudadanía ha distinguido dos procesos dinámicos expresados en las lógicas sociales de ampliación de la ciudadanía: la inclusión de personas como ciudadanos y la expansión o creación de derechos (Dalton, 2006; Marshall, 1997). Respecto de la inclusión, contempla tanto una dimensión de incorporación cuantitativa de individuos, como una de inclusión cualitativa de tipos de personas y grupos a quienes antes no se confería ciudadanía (Dahl, 1989; Wallerstein, 2003). Se han identificado procesos de inclusión de proletarios, mujeres, grupos raciales y étnicos, y una variedad de grupos desventajados (Isin y Wood, 1999; Kymlicka y Norman, 1995; Mouffe, 1992; Wallerstein, 2003). Sobre el caso chileno, los pocos estudios que conocemos (Barros y Vergara, 1991; Maza, 1995; Valenzuela, 1985) identifican algunos de los patrones de inclusión que también han sido descritos en la literatura clásica sobre el tema (Dahl, 1989; Marshall, 1997; Moore, 1969).

Sobre la expansión de los derechos, el clásico estudio de T. H. Marshall (1997) es considerado representativo de una comprensión estrecha y minimalista. No obstante, la suya no es una concepción sedimentada e inamovible de ciudadanía, sino una más bien dinámica. Para él, las aspiraciones sociales que se dirigen al ideal contemplan una medida más completa de la igualdad, un enriquecimiento del contenido de la ciudadanía y del canon de derechos y deberes, y un aumento del número de ciudadanos plenos. Por ello, no es casual que su teoría haya sido un antecedente valorado por estudios posteriores que enfatizan la necesidad de ampliar la intensión del concepto de ciudadanía más allá de la esfera de la política institucional, el Estado y de los llamados derechos civiles, políticos y sociales (Isin y Turner, 2002; Isin y Wood, 1999; Mouffe, 1992; Nyers, 2007; Pinto y Flisfisch, 2011). Así, se ha sugerido incluir los derechos culturales (Miller, 2002; 2011; Stevenson (ed.), 2001); los derechos de grupos (Cuevas, 2013; Dhamoon, 2009; Isin, 2009; Kymlicka y Norman, 1995; Young, 1990); los derechos de pueblos originarios o indígenas (Bello, 2004; Ivison et al. (eds.), 2000) los derechos asociados a la sexualidad y la pluralidad de formas de vida íntima (Plummer, 2003); los derechos medioambientales y la ciudadanía ecológica (Dobson, 2003; Isin y Wood, 1999); y el derecho a la ciudad (Lefebvre, 1996; Harvey, 2008), entre otros.

Como consecuencia de tales consideraciones, teóricas o de procesos sociales (demandas de los movimientos sociales y los cambios culturales globales), diferentes autores propician una concepción extendida de la 
ciudadanía, que goza hoy de cierta aceptación en las ciencias sociales (con excepción tal vez de la ciencia política) (Isin y Wood, 1999; Turner, 1990; Isin y Turner, 2007). Ahora bien, más allá de tales consideraciones, parece recomendable estudiar las modalidades propias de desarrollo de la ciudadanía con sus avances y retrocesos en procesos sociales situados en sociedades del Sur Global, con especial atención en América Latina y Chile (Pinto y Flisfisch, 2011; Oxhorn, 2004; van Der Ree, 2011). Por ejemplo, cabe preguntarse por el efecto de las dictaduras en la región, en tanto retroceso en los derechos políticos y civiles; o por los efectos de las políticas de ajuste económico y re-estructuración económica y social impulsadas por los proyectos neoliberales en la región, que afectaron negativamente los derechos sociales y culturales de clases sociales populares y grupos subalternos (Dagnino, Olvera y Panfichi, 2006). Son principalmente las secuelas de la implementación de los proyectos neoliberales en la región para la ciudadanía los que se discuten en este escrito, lo que implica profundizar en el significante "neoliberalismo"

\section{El neoliberalismoy los procesos de neoliberalización.}

La extensa literatura que discute el neoliberalismo presenta varias aproximaciones al fenómeno. Mientras algunos destacan la dimensión ideológica en tanto fundamentalismo de mercado (Block y Somers, 2014), otros enfatizan su carácter de proyecto político orquestado desde el Estado (Wacquant, 2012). Mientras unos destacan su lógica gubernamental como racionalidad economicista generalizada a la sociedad, las personas y el Estado (Brown, 2015), otros subrayan el carácter multifacético de sus políticas y su diseño institucional, que es sectorialmente heterogéneo (Maillet, 2015). En este sentido, por ejemplo,Brenner et al. (2010), han propuesto usar el término neoliberalización en desmedro del término neoliberalismo para enfatizar el fenómeno en tanto que proceso concreto (el neoliberalismo realmente existente) y no como pura doctrina. En su perspectiva, más que una ideología abstracta con un conjunto de valores e ideas, la neoliberalización es la instauración de un proyecto variopinto cuya constante parece ser su inclinación por los intereses del sector de negocios. Siguiendo esta línea, se ha concebido el neoliberalismo como un fenómeno operando en al menos tres niveles: un hito histórico marcado por el ascenso de la teoría monetarista como eje directriz de la gobernanza económica y social; un set de políticas públicas que basan la provisión de servicios públicos en mecanismos de mercado; y una ideología que postula la libertad individual -expresada en el mercado- como máximo valor humano (Fridman 2017).

Se puede señalar entonces, que el neoliberalismo implica un complejo ensamblaje de ideas, racionalidad política, procesos y prácticas que, por una parte, intensifican la mercantilización de variadas esferas de la vida (sociedad y naturaleza), y produce una desigual regulación de la economía por medio de intervenciones del Estado, (cfr. Brenner et al 2010, Brown 2015, Wacquant, 2012). Esto nos obliga a repensar: 1) la posición y modo de 
intervención del Estado en su relación con el Mercado y la Sociedad, 2) la formación de las intervenciones del Estado neoliberal, que pueden ir desde la prescindencia y el retroceso del sector público, a un rol de planificador estratégico sectorial.

En general, los estudios sobre neoliberalismo han contravenido la idea común de que este es un sistema monolítico y han planteado que existen "variedades" de neoliberalismos, concebidos a partir de las distintas modalidades con que los Estados se relacionan con los mercados (Maillet, 2015). Del mismo modo, mientras la versión ortodoxa del neoliberalismo sugiere que el rol del Estado ha de reducirse a desregular, la investigación en economía política y sociología económica ha demostrado que los Estados tienen un rol activo en la producción y proliferación de los mecanismos de mercado propios del neoliberalismo, y con ello en el formateo de los ciudadanos mismos. Los Estados generan condiciones institucionales para que se produzcan las dinámicas de competencia propia de los mercados (Fligstein, 2001; Hernández, 2017), tales como leyes anti-monopolios y convenciones, así como subsidios a la demanda.

Del mismo modo, operando bajo el paradigma neoliberal según el cual los problemas públicos requieren soluciones de mercado, los Estados también se convierten en "jugadores” dentro de los propios mercados para competir por la provisión de servicios a sus ciudadanos (Maillet, 2015). En muchos casos, como ha mostrado Mazzucato (2014), ha sido el Estado el que ha realizado importantes inversiones en investigación y el desarrollo de tecnologías de punta, que después son apropiadas material y discursivamente por sectores empresariales que buscan expandir los mercados.

Finalmente, los Estados también crean instrumentos de política pública para que los individuos puedan entenderse a sí mismos como actores racionales u homo economicus, tales como políticas de educación financiera que "formatean" a los ciudadanos conforme a principios de calculabilidad(Callon y Caliskan 2009; Marron, 2013) y marcos morales (Marambio en este número). Punto que introduce notaciones políticas específicas sobre la relación entre neoliberalización y ciudadanía.

Llevándolo al plano político, la relación entre neoliberalismo y ciudadanía puede entenderse desde la clave de lectura de la economía política. En primer lugar, esto implica reconocer que, tal como avanzaba Karl Polanyi en "La Gran Transformación”, la expansión de los mercados conlleva una reacción de la sociedad por protegerse, y esta puede tomar la forma tanto de movimientos sociales como de esfuerzos reaccionarios neo-conservadores (Polanyi 1945). Los movimientos sociales, por su lado, no solo buscan promover o reconquistar derechos sociales, sino también disputar los efectos distributivos de los mercados (Beckert, 2013). De paso, enfrentan desafíos que no enfrentaron sus homólogos durante la primera expansión del liberalismo. Por un lado, la mercantilización de la naturaleza parece presentar límites estructurales que amenazan la subsistencia del ecosistema mismo. 
Por otro lado, la consolidación del “capitalismo de plataforma” -impulsado por empresas de alcance global como Google, Facebook o Amazon- ha trasladado la disputa por los derechos sociales a nuevos terrenos, tales como la de los derechos de propiedad sobre la información privada o la activación de procesos de creación destructiva(Srnicek, 2017).

Por eso, si Foucault (2008) definía lagubernamentalidad neoliberalen función la lógica probabilística propia del capitalismo de la segunda mitad del siglo XX , la consolidación del capitalismo de plataformainvita a pensar las nuevas formas de subjetivación, asociadas al ascenso de los algoritmos, el internet de las cosas y el Big Data como técnicas de control.

Por otro lado, las reacciones neo-conservadoras a la excrecencia de los mercados en el neoliberalismo, amenazan con poner un freno a las disputas por el reconocimiento y extensión de derechos de ciudadanía a trabajadores migrantes o minorías sexuales (a la vez que comparten la inclinación neoliberal de bloquear cualquier intento de imponer impuestos al 1\% más rico) (Crouch, della Porta y Streeck, 2016). Esta reacción a las fuerzas globales de la liberalización económica, cuya manifestación son los renovados populismos de derecha, implica una reconfiguración, y reajuste, de los derechos de ciudadanía que habrían logrado los nuevos movimientos sociales e invita a pensar la ciudadanía en el contexto de una lucha cultural con renovado énfasis conservador.

Finalmente, el neoliberalismo debe entenderse como una reconfiguración del orden socio- económico que determina las posibles reconfiguraciones de la ciudadanía en contextos democráticos. En este sentido se entiende al neoliberalismo como una arremetida del capital contra los controles institucionales que caracterizaron el orden keynesiano (Europa) o desarrollista (Latinoamérica) de la post-guerra y que se impusieron sobre los principios de eficiencia económica a favor del principio de justicia social - trabajo organizado, protección social y regulación de los mercados financieros (Streeck, 2014). Con más de 30 años en vigor, esta arremetida ha producido una situación marcada por la excesiva concentración del ingreso en el $1 \%$ más rico de la población y niveles de desigualdad que retornan a los de inicios del siglo XX (Piketty, 2014); acumulación de poder en grandes corporaciones y acreedores internacionales cuyas fortunas crecen cuando el resto del mundo experimenta estagnación (Crouch, della Porta y Streeck, 2016); una alta movilidad de los factores productivos promovida por la libertad de comercio que es utilizada por el capital herramienta de disuasión y presión para imponer sus intereses por sobre los demás, conocida como "huelga Kaleckiana” (Fairfield, 2015); y una capacidad ampliada de las elites de imponer disciplina fiscal y de hacer a otros para pagar los costos de sus malas decisiones mediante la socialización de las pérdidas, como bien mostró la crisis de 2008 (Crouch, della Porta y Streeck, 2016)

Frente a esta arremetida del sector de negocios, los límites de la ciudadanía se re-dibujan en contextos postdemocráticos (Crouch, 2004), caracterizado por una situación en la cual las instituciones democráticas 
liberales -elecciones regulares, orden de la ley- están en pleno rigor, pero las decisiones que tienen efectos distributivos no están al alcance de los ciudadanos . Estos controles se encuentran más bien relegados a las cúpulas de técnicos -principalmente economistas, bancos centrales y organismos supra-nacionales- que comulgan la fe en la auto-regulación de los mercados y privilegian los principios de eficiencia económica por sobre los de justicia social. La post-democracia conduce así a una creciente fusión entre el poder económico con el político, reforzando su oligarquización, en la cual el destino de las elites parece desacoplarse del de los ciudadanos de los cuales extraen su riqueza.

\section{Neoliberalización y ciudadanías en el Sur.}

Todo lo referido hasta acá es del orden de lo cotidiano a escala global en la actualidad. No obstante, mientras algunas sociedades de la vieja Europa recientemente experimentan las políticas de austeridad (Della Porta y Matonni, 2014; Della Porta, 2015), otras sociedades de la periferia europea, como España, Portugal y Grecia, ya la han incorporado en su funcionamiento diario, objetivando sus alcances (Santos, 2016). Por su parte, en las sociedades de América Latina o África presentan tal rasgo de manera más naturalizada o normal (Santos \& Meneses, 2014; Gaudichard, 2012; Svampa, 2015), dejando ver en tal rasgo su condición periférica y subalterna. Por ejemplo, en Chile el laboratorio neoliberal implementado después de 1973 (Gárate, 2012; Gaudichaud, 2015), ha continuado impulsando procesos de neoliberalización hasta la actualidad como puede verse en el caso del derecho a la educación superior después del retorno de la democracia, reducido a su definición como bien de consumo (Cuevas \& Paredes, 2018; Donoso, 2017).

Tal condición de subalternidad, nos permite hacer referencias a los procesos radicales de neoliberalización que vivencian las sociedades de las periferias del globo, así como las diferentes expresiones de ciudadanía en las sociedades del Sur Global. El “Sur”, en la propuesta acá presentada, no se refiere a un concepto geográfico, aunque se superpone a él. Es más bien a una metáfora de las relaciones sociales subalternas, desiguales y de exclusión que ha producido el desarrollo del capitalismo, y sus relaciones con el colonialismo y el patriarcado, a nivel global. Estas relaciones desiguales se han visto radicalizadas en contextos geográficos donde se experimentan procesos vivos deneoliberalización (Santos \& Meneses, 2014). Entonces, con la expresión "Sur Global" se trata de dar cuenta de la diversidad de experiencias y expresiones en las que se configuran las formas de ciudadanía en respuesta a los procesos de neoliberalización de nuestras sociedades, así como de las tensiones posibles de observar entre ciudadanías y neoliberalización, que implican de por sí una preocupación por la democracia y las dificultades que acarrea dicha tensión para su consolidación y permanencia (Santos, 2016; De la Cuadra y Paredes, 2017).

Los diferentes artículos de esta sección se hacen cargo de las complejas relaciones entre los neoliberalismos y las ciudadanías realmente exis- 
tentes en contextos del Sur. Un mérito de los trabajos recopilados es que abordan los arreglos concretos y las racionalidades aplicadas en diferentes ámbitos (opinión pública de la ciudadanía y autonomía indígena, endeudamiento), sectores de política (política sociales) y escalas (nacional, local y barrial). Los artículos analizan críticamente las diferentes (des)articulaciones de la ciudadanía con el neoliberalismo, sin caer en el facilismo de la impugnación de la ciudadanía privatizada y mercantilizada. En efecto, varios de los artículos exploran la formación del ciudadano concreto en la época de la hegemonía neoliberal; los efectos moralizantes de los ideales neoliberales y cómo contribuyen a la constitución de una forma particular y contingente de sujeto político (ciudadano); las políticas públicas neoliberal y su efecto constituyente. Pero también se estudian los efectos menos obvios, como las políticas de resistencia de los actores, para quienes la ciudadanía se convierte en un refugio desde el cual confrontar los embates de las políticas neoliberales. Los estudios muestran cómo al estudiar políticas sectoriales específicas o realidades de escala local, la realidad observada se distancia de los lineamientos estilizados de las ideologías, las teorías y las doctrinas, o al menos muestra desajustes respecto de ellas. Si bien el conjunto de artículos de este dossier es más bien heterogéneo, su lectura y consideración como conjunto sugiere la existencia de una multiplicidad de arreglos sociopolíticos y económicos posibles, en los que los contornos de la ciudadanía y el neoliberalismo se afectan mutuamente, redefiniendo así las posibilidades de una y otra categoría para iluminar la realidad.

El primer artículo de la sección, de autoría de Juan Esteban Fernández y Claudio Fuentes S., se titula Percepciones sociales sobre el derecho de autonomía de los pueblos indígenas en Chile. En élse estudian estadísticamente las actitudes sociales respecto al derecho de autonomía de los pueblos indígenas en Chile y se explora el perfil de quienes favorecen/rechazan la autonomía indígena. El estudio concluye que la demanda por autonomía indígena tiende a ser más relevante entre personas que se autoidentifican como indígenas, jóvenes, con niveles de escolaridad baja, que viven en zonas urbanas, de izquierda, y de la macrozona centro y norte.

\section{El artículo titulado $\dot{i}$ Nuevas territorialidades vecinales en el Chile} neoliberal? de Letelier Troncoso et alt., establece una vinculación interesante entre las formas estatales de intervención territorial y ciertas modalidades en las que ciudadanos entretejen lazos con otros para redefinir a un actor social (el vecino) y al espacio asociativo local (lo vecinal). El texto no niega la crisis por la que atraviesan las organizaciones vecinales como espacios de participación, en Chile, pero sabe que son justamente estos espacios uno de los terrenos de mayor fertilidad para fomentar una ciudadanía alternativa a la forma neoliberal individualizada. Los autores/as, encontraron en la experiencia vecinal concreta y sus prácticas relacionales, algunas claves para entender, de manera incipiente y expresadas como tendencias posibles más que existencias, la amplitud de la noción de ciudadanía, no reducida a la membresía de una comunidad societal de carácter nacional. Es en el cambio de escala, lo vecinal, en que se observa una comprensión mayor y un ejercicio más pleno de los derechos vinculados al 
habitar, en base a procesos de autogestión vecinal y de relación más productiva con la institucionalidad local, implicando un cambio en la situación de poder de los actores vecinales respecto al espacio que habitan. Un corolario de estas relaciones incipientes es que desnudan un conjunto de problemas relacionados con la institucionalidad política y la política urbana. La idea del derecho a la ciudad, con toda su impronta anti-neoliberal, vía actos y prácticas de una ciudadanía vecinal, resuena como música de fondo en el escrito.

Por su parte, la contribución de MarambioEndeudamiento "saludable", empoderamiento y control social aborda la idea de "endeudamiento saludable" y el derecho a pagar en Chile operan como mecanismos de gubernamentalidad. A pesar de que estas actividades son presentadas al público como formas de empoderamiento social, Marambio utiliza entrevistas con reguladores y consumidores para mostrar que finalmente operan como formas de control social. De este modo, muestra cómo una idea transversal a las políticas de educación financiera (de que hay una forma sana de endeudarse) se utiliza como una imagen sobre la que se proyecta una noción normativa del "ciudadano promedio". Esta forma de ciudadanía contenida en los instrumentos financieros se caracteriza por transferir la responsabilidad al sujeto y a los mecanismos de auto-control que Foucault denominó como propios de la gubernamentalidad neoliberal. En tanto dispositivo de subjetivación, el endeudamiento saludable aparece entonces como una herramienta para ganar control sobre la vida financiera de las personas, y como tal constituye lo que Miller and Rose (1990) llaman un dispositivo de "gobierno a distancia", a través del cual se performa el ciudadano neoliberal.

El artículo de Ana Natalucci El neoliberalismo en acto: políticas sociales y experiencias organizativas en Argentina (2009-2016) muestra cómo es que una política pública con lógicas neoliberales en Argentina (PRIST), produce las condiciones para la emergencia de una organización sindical nacional (CTEP) que otorga reconocimiento a los trabajadores informales. De esta forma, muestra el fenómeno contra-intuitivo de que las políticas públicas neoliberales pueden abrir un campo de acción en el cual los actores sociales no necesariamente pierden su capacidad de transformación y agenciamiento. A diferencia de lo que comúnmente se afirma, las políticas neoliberales también pueden tener como consecuencias no intencionadas el producir condiciones para la emergencia de formas de asociación social que convencionalmente se entienden como opuestos al neoliberalismo. De esta manera, un agenciamiento surgido de una política neoliberal -como la organización sindical nacional (CTEP)- termina constituyendo un mecanismo de extensión de ciudadanía por medio de la inclusión de trabajadores informales al dominio de las políticas públicas redistributivas.

Finalmente, el artículo de Carolina Rojas Lash titulado Afecto y cuidado: pilar de la política social neoliberal sostiene que en Chile se instalaron tecnologías de subjetivación y de gobierno que, aunque se orientan a 
proteger a los individuos y promueven formas de intervención social que moviliza afecto y se orienta a cuidar, éstas terminan por articularse con la racionalidad neoliberal para, finalmente, reforzarla. De este modo, la política social contemporánea produce una nueva relación entre ciudadanos y Estado, que en lugar de distanciarse del orden neoliberal, lo sostiene. 


\section{Nota}

${ }^{1}$ En efecto, existen casos de coaliciones y partidos conservadores, y otros con bases de apoyo progresistas, cuyas políticas concretas no difieren en algunos de sus principios fundamentales y que han sido blanco del mismo ciclo de contestación polìtica y protesta (cf. Gudynas 2018). 


\section{Referencias}

Aristóteles (1988) Política. Madrid, España:Gredos.

Aljovin de Losada, C. (2009). Ciudadano y vecino en Iberoamérica, 17501850: Monarquía o República. En Javier Fernández Sebastián (ed.) Diccionario político y social del mundo iberoamericano. La era de las revoluciones, 1750-1850. Madrid, España: Fundación Carolina.

Ancelovici, M., Pascale, D., \&Heloise, N.(2016). Thestreetpolitics in theausterityage. Fromthe indignados to occupy. Amsterdam, Países Bajos: AmsterdamUniversityPress.

Barros, L. y Vergara, X. (1991). Los grandes rasgos de la evolución del Estado en Chile: 1826-1925. En Atria, R. y Tagle, M. (eds.). Estado y política en Chile: ensayos sobre las bases sociales del desarrollo político chileno.(pp. 81 -124). Santiago, Chile: CPU,

Bauman, Z. (1991). Modernity and Ambivalence. Nueva York, Estados Unidos:CornellUniversityPress.

Beckert, J.(2013). Capitalism as a System of Expectations. Politics\&Society, 41(3), 323-350.

Barbalet, J. (2007). “Citizenship”. En G. Ritzer (ed.) TheBlackwellEncyclopedia of Sociology. (pp. 497-500). Oxford, Inglaterra:Blackwell,

Bellei, C., Cabalín, C., y Orellana, V. (2014). The 2011 Chilean studentmovementagainst neoliberal educationalpolicies. Studies in HigherEducation, 39 (3), 426 - 440. http://dx.doi.org/10.1080/ 03075079.2014.896179

Bringel, B., y Pleyers, G. (eds.). (2017). Protestas e indignación. Los movimientos sociales en el nuevo orden mundial. Buenos Aires, Argentina: Clacso.

Budrovich, J., y Cuevas, H., (en prensa) Contestedlogistics? Neoliberal modernization and resistance in theportcity of Valparaíso. En Ness, I. y Wilson, J. (eds.) ChokePoints: LogisticsWorkersDisruptingthe Global SupplyChain. Londres, Inglaterra: Pluto Press.

Çalýpkan, K., \&Callon, M. (2009). Economization, part 1: shifting attention from the economy to wards processes of economization. Economy and society, (38) 3, 369-398.

Cuadra, X. (2014). Nuevas estrategias de los movimientos indígenas contra el extractivismo en Chile, Revista CIDOB d'AfersInternacionals, 105, 141-163. 
Crouch, C. (2004). Post-democracy. Cambridge, Inglaterra: PolityPress.

Crouch, C., Della Porta, D., y Streeck, W. (2016). Democracy in neoliberalism? AnthropologicalTheory, 16(4), 497-512.

Cuevas, H., Dasten, J., y Rojas, J. (eds.) (en prensa) Capitalismo Global en América Latina. Concepción, Chile: Universidad de Concepción-RIL.

Cuevas, H., y Paredes, J.P. (2018). Esfera pública, actos de ciudadanía y arenas públicas: la redefinición de la educación del espacio público por las protestas estudiantiles en Santiago. En Del Valle, N. (ed.) Transformaciones de la esfera pública en el Chile neoliberal. Luchas sociales, espacio público y pluralismo informativo, (pp. 39-68). Santiago, Chile: RIL Editores

Dagnino, E., Olvera, F. y Panfichi, A. (Coords). (2006). La disputa por la construcción democrática en Amèrica Latina. México D. F., México: FCE/CIESAS.

Dahl, R. (1989). La Poliarquía. Madrid, España: Tecnos.

Dalton, R. (2006). CitizenPolitics: PublicOpinion and PoliticalParties in Advanced Western Democracies. Chatham, NJ: Chatham House.

De la Cuadra, F.y Paredes, J.P.(2017). Las amenazas y desafíos de la Democracia. Polis, Revista Latinoamericana(46), 5-16. Recuperado de https://journals.openedition.org/polis/12416

Della Porta, D. (2015). Social movements in times of theausterity. Cambridge; Malden, Estados Unidos: Politypress.

Della Porta, D.\&Mattoni, A. (Eds). (2014). Spreadingprotest. Social movements in time of crisis. Essex, Inglaterra: ECPR Press.

DisiPavlic, R. (2018). Sentenced to Debt: ExplainingStudentMobilization in Chile. Latin American ResearchReview, 53(3)

Donoso, S. (2017). Outsiders and Insidersstrategies: Chile's students movements, 1990-2014. En Von Bülow, M. y Donoso, S. (Eds). Social movements in Chile. Organization, trajectories \& politicalconsequences (pp. 65-97) .New York, Estados Unidos: PalgraveMacmillan.

Errejón, I. \&Mouffe, Ch. (2015). Construir pueblo. Hegemonía y radicalización de la democracia. Barcelona, España: Icaria.

Escobar, A. (2003). “OtherWorlds Are (already) Possible: CyberInternationalism and Post-Capitalist Cultures.” Presented at Cyberspace Panel, LifeafterCapitalismProgramme, World Social 
Forum, Porto Alegre, January 23-28, 2003. At: http://www.zmag.org/ lac.htm

Escobar, A. (2010). Una minga para el postdesarrollo: lugar, medio ambiente y movimientos sociales en las transformaciones globales. Lima, Perú: Programa Democracia y Transformación Global Universidad Nacional Mayor de San Marcos /Facultad de Ciencias Sociales.

Fairfield, T.(2015). Structuralpower in comparativepoliticaleconomy: perspectivesfrompolicyformulation in LatinAmerica. Business and Politics, 17(3), 411-441.

Fleet, N. y Guzmán, C. (2016). MassHigherEducation and the 2011 StudentMovement in Chile: Material and IdeologicalImplications. Bulletin of LatinAmericaResearch 36 (2), 160-176. https://doi.org/ 10.1111/blar.12471

Foucault, M. (2008). TheBirth of Biopolitics. Lectures at theCollége de France 1978-1978. Basingstoke, Reino Unido:PalgraveMacmillan.

Forment, C. (2003). Democracy in LatinAmerica 1760-1900. Vol. 1. CivicSelfhood and PublicLife in Mexico and Perú.Chicago, Estados Unidos: University of Chicago Press

Fridman, D. (2017). FreedomfromWork. EmbracingFinancialSelf-Help in theUnitedStates and Argentina. California, Estados Unidos: Stanford UniversityPress.

Gamboa, R. (2011). Reformando Reglas Electorales: La Cédula Única y los Pactos Electorales enChile (1958 -1962) Revista de Ciencia Política 31 (2): 159-186.

Gárate, M.(2012). La revolución capitalista de Chile (1973-2003). Santiago, Chile: UAH.

Garcés, M. (2012). El despertar de la Sociedad. Los movimientos sociales en Amèrica Latina y Chile. Santiago, Chile: Lom.

García Linera, Á. (2008). La potencia plebeya. Acción colectiva e identidades indígenas, obreras y populares en Bolivia. Buenos Aires, Argentina: Clacso/Prometeo.

Gaudichaud, F. (2016). La vía chilena al neoliberalismo. Miradas cruzadas sobre un país laboratorio. Revista Divergencias. 5 (6), 13-28.

Gaudichaud, F. (Ed.). (2012). El volcán latinoamericano. Izquierdas, movimientos sociales y neoliberalismo. Concepción, Chile: Escaparate 
Gerring, J. y Barresi, P. (2003). PuttingOrdinaryLanguage to Work. A minmaxStrategy of Concept Formation in the Social Sciences. Journal of TheoreticalPolitics 15 (2) 201-232.

Grez Toso, S. (1997). La cuestión social en Chile. Ideas y debates precursores (1804-1902). Santiago, Chile: DIBAM.

Guerra, F.X. (1992). Modernidad e independencias: ensayos sobre las revoluciones hispánicas. Madrid, España: Mapfre.

Gudynas, E. (en prensa). Disputas entre variedades de desarrollo y el cuadrilema de la globalización.En H. Cuevas, D. Julián y J. Rojas (eds.) Capitalismo Global en América Latina. Concepción, Chile: Universidad de Concepción-RIL.

Hale, C. (2007). Más que un indio. Ambivalencia racial y multiculturalismo neoliberal en Guatemala. Guatemala, Guatemala: AVANCSO.

Hartlyn, J. y Valenzuela, A.(1997). La democracia en América Latina desde 1930 y Una nota sobre la ciudadanía. En Historia de América latina: Política y sociedad desde 1930. (Vol.12).(pp.11-72).Barcelona, España: CRÍTICA Grijalbo Mondadori,

Hernández, J. (2017). Sociología del Mercado en América Latina: Hacia una agenda de investigación. Sociológica(91), 1-35.

Isin, E. y Turner, B.S. (eds.) (2002). Handbook of CitizenshipStudies, London, Inglaterra:Sage.

Isin, E. y Turner, B. S. (2007) InvestigatingCitizenship: An Agenda forCitizenshipStudies, CitizenshipStudies 11(1):5-17.

Isin, E. y Wood, P. (1999). Citizenship and Identity. Londres. Inglaterra: Sage.

Janoski, Th. (1998). Citizenship and Civil Society. Cambridge, Inglaterra: CUP.

Janoski, Th., Alford, R., Hicks, A., y Schwartz, M. (Eds.). (2005). TheHandbook of PoliticalSociology: States, Civil Societies, and Globalization. Cambridge, Inglaterra: Cambridge UniversityPress.

Kant, I. (1994). Respuesta a la pregunta: ¿qué es la ilustración?, Revista Colombiana de Psicología, (3)7-10 U. Recuperado de https:// revistas.unal.edu.co/index.php/psicologia/article/view/15803/16619

Kymlicka, W. \& Norman, W. J. (1994). Return of theCitizen, Ethics, 104, (2), 352-381. 
Klingemann, H, y Fuchs, D. (eds.). (1995). Citizens and theState. Oxford, Inglaterra:OUP.

Maillet, A. (2015). Variedades de neoliberalismo. Innovación conceptual para el análisis del rol del Estado en los mercados. Revista de Estudios Políticos, 169, 109-136.

Maza, E. (1995). Catolicismo, anticlericalismo y La extensión del sufragio a la mujer en Chile, Revista de Estudios Públicos 58, 137-197.

Marron, D. (2013). Informed, educated and more confident": financialcapability and theproblematization of personal financeconsumption. ConsumptionMarkets\& Culture, 1-21.

Marx, K. (1978). La cuestión judía. En Manuscritos de París. Escritos de los “Anuarios francoalemanes”(1844). Barcelona, España: Crítica / Grijalbo, 178-208.

Mazzucato, M. (2014). Theentrepreneurialstate: debunkingpublic vs. private sector myths. London, Inglaterra: AnthemPress.

Millar, R. (1982). La elección presidencial de 1920: tendencias y prácticas políticas en el Chile parlamentario. Santiago, Chile: Editorial Universitaria.

Moore, B. (1969). Social Origins of Dictatorship and Democracy: Lord and Peasant In theMaking of theModern World. Boston, Estados Unidos: BeaconPress

Moulian, T. (1993). La forja de ilusiones: el sistema de partidos 19321973. Santiago, Chile: Univ. ARCIS / FLACSO.

Piketty, Th. (2014). Capital in thetwenty-firstcentury. Cambridge, Estados Unidos: BelknapPress of Harvard Univ. Press.

Polanyi, K. (1945). La gran transformación. México D.F, México: Fondo de Cultura Económica.

Ramírez, J. (2016). Hacia el Sur. La construcción de la ciudadanía suramericana y la movilidad intrarregional. Quito, Ecuador: CELAG.

Richards, P. (2013). Race and theChileanMiracle. Neoliberalism, Democracy and theIndigenousRights. Pittsburgh, Estados Unidos: Pittsburgh UniversityPress.

Santos, B. S. y Meneses, M. P. (Eds). (2014). Epistemologías del Sur (perspectivas). Madrid, España: Akal. 
Santos, B. S. y Rodríguez-Garavito, C. (2007). El derecho y la globalización desde abajo. Barcelona, España: Anthropos-UAM.

Santos, B. S. (2016). Democracia al borde del caos. México D.F, México: Siglo XXI.

Santos, B. S. (2003). TheWorld Social Forum: Toward a CounterHegemonicGlobalization. Presentado en: XXIV International Congress, Latin American StudiesAssociation, LASA, Dallas, marzo 27-29. http://www.ces.fe.uc.pt/bss/fsm.php

Santos, B. S. (2002). Towards a New Legal CommonSense. London, Inglaterra: Butterworth.

Sassen S. (2015) Incompletud y la posibilidad de hacer. ¿hacia una ciudadanía desnacionalizada? En Revista Mexicana de Ciencias Políticas y Sociales. UNAM,(226) 107-140.

Srnicek, N. (2017). Platformcapitalism. Cambridge, Inglaterra: PolityPress.

Schmalz, S. y Ramírez, M. (eds.). (2018). Extractivismo en Sudamérica después del auge de las materiasprimas.Buenos Aires, Argentina: Editorial Biblos.

Streeck, W. (2014). Buying time: thedelayed crisis of democraticcapitalism. London, Inglaterra: Verso.

Streeck, W. (2016). Howwillcapitalismend? Essayson a failingsystem. London, Inglaterra: Verso.

Stuven, A.M.(2000). La Seducción de un Orden: Las elites y la construcción de Chile en las polémicas culturales y políticas del Siglo XIX. Santiago, Chile :Ediciones Universidad Católica de Chile.

Svampa, M. (2015). Commodities Consensus: Neoextractivism and Enclosure of theCommons in LatinAmerica, South AtlanticQuarterly 114 (1): 65-82. Recuperado de https://doi.org/10.1215/00382876-2831290

Valenzuela, A.\&Valenzuela, S. (1983). Los Orígenes de la Democracia. Reflexiones Teóricas sobre el Caso de Chile, Revista de Estudios Públi$\cos 12:$ 7-39. 